\title{
Impacto económico en el medio odontológico durante la pandemia del COVID-19: revisión integradora.
}

\section{Economic impact in the dental field during COVID-19 pandemic: integrative review.}

\author{
Francisco Cázares-de León,* María Gabriela Peraldi-Sada, ${ }^{\ddagger}$ Luis Daniel Aneyba-López, ${ }^{\S}$ David Ernesto Soto-Gámez ${ }^{\natural}$
}

\section{RESUMEN}

La pandemia por COVID-19 no sólo ha generado un impacto negativo en la salud, sino que la economía global también se ha visto mermada, afectando más a los países subdesarrollados. Con relación a estos daños en las finanzas de los profesionales de la salud, existen algunos efectos que derivan de la pandemia COVID-19, los cuales tienen una fuerte repercusión en la economía de todos los trabajadores a nivel mundial y el ámbito odontológico no es la excepción. Esta revisión se obtuvo mediante la búsqueda de la información en una exploración electrónica en las bases de datos PubMed, Cochrane Library, LILACS, SciELO y Latindex. El impacto económico derivado de esta pandemia, sin lugar a dudas, ha afectado la economía de los odontólogos de práctica pública y privada, por lo que la toma de decisiones en la odontología debe contemplar un uso equilibrado de los recursos financieros.

Palabras clave: Pandemia, impacto económico, odontología, economía, COVID-19, salud bucal.

\section{ABSTRACT}

The COVID-19 pandemic has not only generated a negative impact on health, but the global economy has also been reduced, being the underdeveloped countries the most affected ones. In relation to these damages in the finances of health professionals, there are some effects that derive from the COVID-19 pandemic, having a strong impact on the economy of all workers worldwide and the dental field is no exception. This review was obtained by searching the information through an electronic examination in databases like PubMed, Cochrane Library, LILACS, SciELO and Latindex databases. The economic impact derived from this pandemic has undoubtedly affected the economy of dentists in public and private practice, so that decision-making in dentistry must consider a balanced use of financial resources.

Keywords: Pandemic, economic impact, odontology, economy, COVID-19, oral health.

\section{INTRODUCCIÓN}

$\mathrm{L}$ a reciente aparición del síndrome respiratorio agudo severo coronavirus 2 (SARS-CoV-2) y su asociación con ciertas comorbilidades, ha generado en la comunidad internacional, en todos los aspectos, un impacto a nivel de salud pública por la cantidad de muertes, hasta la fecha van más de 75 millones de casos confirmados y más de 1 millón 690 mil muertes a nivel mundial, según datos de la Organización Mundial de la Salud (OMS). En México, hay más de 1 millón 300 mil casos confirmados y más de 117 mil muertes; mientras que Perú cuenta con más de 1 millón y 37 mil, respectivamente. Como es de esperarse, estos datos epidemiológicos no sólo han generado un impacto negativo en la salud, sino también en la economía global, lo que afecta más a los países subdesarrollados. Cabe mencionar que los odontólogos son una población que puede sufrir un máximo riesgo

\footnotetext{
* Doctorado en Filosofía con orientación en Psicología. Universidad de Monterrey (UDEM). Monterrey, México. ORCID: 0000-0002-0481-8956.

¥ Médico Cirujano Dentista. Universidad de Monterrey (UDEM). Monterrey, México. ORCID: 0000-0001-7117-9050.

§ Maestría en Innovación de Negocios. SLEEPADENT. Monterrey, México. ORCID: 0000-0002-2476-6356.

" Maestría en Gestión Directiva en Salud. Consulta privada. Monterrey, México. ORCID: 0000-0001-8669-6632.

Recibido: 30 de enero de 2021. Aceptado: 01 de febrero de 2021.
}

Citar como: Cázares-de León F, Peraldi-Sada MG, Aneyba-López LD, Soto-Gámez DE. Impacto económico en el medio odontológico durante la pandemia del COVID-19: revisión integradora. Rev ADM. 2021; 78 (1): 42-47. https://dx.doi.org/10.35366/98386

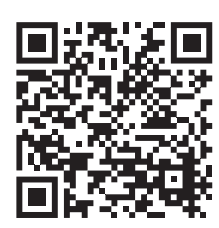


de contagio, pero también enfrentan la posibilidad de que los pacientes no acudan a consulta por el temor a contagiarse dentro del consultorio. Además de esto, los ingresos económicos tanto del odontólogo como de los pacientes, se han visto disminuidos por los efectos negativos que ha causado esta pandemia. ${ }^{1-3}$

El brote inicial del virus inició en un mercado local de mariscos en la ciudad de Wuhan, en China, a finales de diciembre del 2019, y se ha extendido en todo el mundo, lo que ha llegado a ser el mayor desafío de salud pública en las últimas décadas. Por esto, la OMS lo llegó a clasificar como una pandemia a nivel global desde el 11 de marzo del 2020. ${ }^{4-6}$ Por los niveles alarmantes de propagación y gravedad, se ha señalado que es muy importante reforzar las medidas preventivas para evitar que se disemine aún más a nivel masivo, ${ }^{7,8}$ no siendo así con las medidas económicas en un principio.

Este virus, perteneciente a la familia de los Coronaviridae, tiene un periodo de incubación que varía entre los pacientes de 0 a 24 días, según la intensidad de la enfermedad, lo que lo hace aún más peligroso. ${ }^{9-11} \mathrm{La}$ OMS nombró al nuevo virus de neumonía como COVID-19, pero el ICTV (Comité Internacional de Taxonomía de Virus) lo llamó SARS-CoV-2. Como aún no existe un tratamiento de forma definitiva, esto ha generado un desafío para la economía en el Sector Salud. ${ }^{12}$

Estas generalidades son importantes para que el profesional de la salud tenga el conocimiento necesario acerca de esta enfermedad, y pueda comprender que la pandemia COVID-19 debe ser entendida como una problemática de salud pública, y no sólo como una enfermedad respiratoria aislada. Además, el odontólogo juega un rol muy importante en la prevención de la enfermedad, pero también un papel fundamental en la sinergia de la salud con el impacto económico que ésta genera si un paciente se llega a enfermar de manera grave.

La pandemia COVID-19 resulta tener un impacto negativo significativo para la sociedad en su salud y economía, por lo que los desafíos en el manejo de sus efectos son muy densos, resultando ser importantes para los clínicos en el entendimiento de la prevención de esta enfermedad, así como el manejo de los riesgos de la salud. Es muy valioso e importante para entender y comprender la percepción que pueden tener las personas acerca de la necesidad de ir o no ir a consulta, ya que además de temer por su salud, se enfrentan a deficiencias económicas, siendo éstas algunas barreras en el uso de los servicios de salud. ${ }^{1-3}$

Con relación a estos daños en las finanzas de los profesionales de la salud, existen algunos efectos que derivan de la pandemia COVID-19, teniendo una fuerte repercusión en la economía de todos los trabajadores a nivel mundial y el ámbito odontológico no es la excepción, incluso ha sido uno de los más afectados, ya que la odontología ha sido considerada como una de las profesiones con mayor riesgo laboral por el íntimo contacto y relación con la saliva y la sangre, lo cual ha obligado a emplear medidas preventivas muy estrictas $y$ costosas, a esto se añade que durante el inicio del brote de la pandemia sólo se sugería llevar a cabo tratamientos de urgencias. ${ }^{11}$ El nivel de asistencia de los pacientes a consulta también ha sido afectado, reduciendo el número de pacientes tratados, aunque esta cantidad sea diferente en clínicas públicas y privadas. ${ }^{13,14}$

Esta pandemia no sólo ha originado un impacto a nivel de salud pública, además ha causado una gran recesión económica global, Ilevando a gran velocidad hacia una crisis económica inmediata, la cual ha generado pérdida de empleos y continúa amenazando en gran medida la economía global, las sociedades y los derechos humanos..$^{15-17}$ Sin duda, las medidas que se han tomado para mitigar esta pandemia como el confinamiento y aislamiento generalizado de las personas en sus hogares, ha ocasionado una caída en la actividad económica, lo que ha afectado los recursos económicos de los pacientes; específicamente en el sector odontológico ha habido una escasez en la necesidad de ir a buscar atención médicoodontológica, una reducción de proveedores de insumos, encarecimiento en los equipos de protección personal y escasos recursos para tratar a los pacientes. ${ }^{18-20}$ Todo esto está dejando un impacto con saldo negativo en la economía de los profesionales de la odontología. ${ }^{18,19}$

En una encuesta en línea realizada por la Asociación Médica de Texas, en Estados Unidos, se encontró que $68 \%$ del personal de la salud se vio en la necesidad de recortar sus horas laborales debido al COVID-19; 62\% vislumbró una reducción de sus ingresos; así mismo, algunos han declarado tener que clausurar completamente sus consultorios privados. ${ }^{21}$

Al respecto, la mitigación y supresión, necesarias para contener la propagación, han tenido un impacto en la economía, con resultados catastróficos para muchas pequeñas y medianas empresas, después de la implementación de la fase 3 de prevención. La toma de decisiones se ha visto obstaculizada por la falta de datos, falta de información sobre la tasa de letalidad de COVID-19, así como también la contribución a su propagación por parte de los dentistas, ${ }^{9,12}$ por lo que es crucial en estos momentos, para preservar la salud bucal de los pacientes y no afectar más sus finanzas, que a través de los ministe- 
rios de salud y colegios profesionales se tomen medidas de emergencia para ayudar al odontólogo con los altos costos de mantener la seguridad del personal y la de los pacientes en su práctica profesional. ${ }^{15-17}$

La pandemia de COVID-19 ha inducido a una gran recesión económica global, por lo que en estos momentos se está presenciando un escenario llamado «choque de coronavirus», un fenómeno que amenaza con afectar en gran medida la economía, las sociedades y los derechos humanos. ${ }^{18} \mathrm{~A}$ medida que la pandemia se ha expandido y llegado a los países de bajos y medianos ingresos, se han presentado efectos aún más graves debido a que les ha sido más difícil responder ante la pandemia, presentando escasez en la atención médica, menos proveedores, falta de equipo de protección personal y escasos recursos para tratar a los pacientes, lo cual se traduce en una pérdida catastrófica de vidas, así como también en la reducción económica. ${ }^{18-20}$

En torno a esto, el Fondo Monetario Internacional (FMI) ha confirmado que el mundo se enfrentará a su peor recesión económica, teniendo previsto una contracción mundial de 3\% en el año 2020. No se prevé ninguna posibilidad de crecimiento en las perspectivas de la economía mundial, sólo riesgos de regresión, que básicamente consisten en la posibilidad de que la actual pandemia no se pueda controlar o que se repita una situación parecida en 2021, ante esto, la Organización para la Cooperación y el Desarrollo Económicos (OCDE) advierte que, por cada mes de contención, el crecimiento del producto interno bruto (PIB) disminuirá dos puntos porcentuales y ha pedido un «plan Marshall mundial» para contrarrestar los efectos de la pandemia de la COVID-19. ${ }^{20}$

Si bien el impacto económico del COVID-19 aún no es visible al mismo nivel en todos los países, los que están en desarrollo y subdesarrollados, que dependen en gran medida de las cadenas de suministro para sostener su economía, sufren con la posibilidad de afectar a muchos más en un contexto mundial globalizado. ${ }^{19}$

Debido a todos estos antecedentes que ha generado la pandemia del COVID-19 y sus efectos económicos y de salud, es necesario que el odontólogo conozca la trascendencia económica de esta enfermedad, razón principal de este artículo de revisión. Es necesario también que los profesionales de la salud oral entiendan las implicaciones del riesgo de la transmisión del SARS-CoV-2 para comprender mejor el comportamiento de sus pacientes y la enfermedad y así buscar una alternativa efectiva por medio de un equilibrio de bioseguridad y económico, sin dejar de proporcionar una atención odontológica óptima a los pacientes que necesiten de un tratamiento de urgencia dental. ${ }^{22-24}$

\section{MATERIAL Y MÉTODOS}

Estudio retrospectivo de corte transversal llevado a cabo mediante una exploración electrónica para la selección de la información científica en bases de datos PubMed, Cochrane Library, LILACS, SciELO y Latindex. La selección de los artículos se basó principalmente en revisiones de la literatura, investigaciones originales y cartas al editor relacionadas con el impacto económico en salud oral y el COVID-19, en idioma inglés y español del año 2020, considerando la escasa información de literatura científica que existe sobre este tópico.

\section{REVISIÓN DE LA LITERATURA}

Debido a todos los problemas ocasionados por este virus, la OMS ha tomado medidas restrictivas contra la pandemia de COVID-19 en todos los países, ya que ha afectado de manera significativa el entorno laboral, económico y académico. 1,9,25-27

Por el alto nivel de riesgo que existe en los procedimientos odontológicos debido a los bioaerosoles generados durante los procedimientos dentales, que contaminan las superficies en el consultorio dental, pudiendo causar la transmisión del virus a los odontólogos y sus pacientes, ${ }^{9,28,29}$ se requiere de un gasto mayor en equipo de protección personal, lo que encarece los procedimientos odontológicos y, por lo tanto, los costos.

Como el riesgo de contagio en un consultorio dental es alto, ya que el virus se aloja en la saliva, el líquido crevicular y las glándulas salivales, ${ }^{30,31}$ la Asociación Dental Americana (ADA) había recomendado que los odontólogos pospongan tratamientos, y sólo atiendan las urgencias dentales, evitando exponerse a bioaerosoles, tanto ellos como sus pacientes. Al irse incrementando el número de casos en forma exponencial, es indispensable reforzar las medidas de bioseguridad que sean útiles para la práctica odontológica. ${ }^{1,11,32-34}$ Estos intentos por disminuir la probabilidad de contagio por parte de los odontólogos genera mayores costos a los tratamientos dentales, aunado a que la economía de los pacientes se ha visto mermada en esta pandemia, todo esto resulta en un impacto negativo en las finanzas de ambos.

Por esto, muchas han sido las posturas desde el punto de vista de los odontólogos, dueños de las clínicas o consultorios que tienen que adoptar estrictas medidas de seguridad sanitaria para mitigar la pandemia COVID-19, y que tienen que apegarse a los lineamientos impuestos por sus secretarías o ministerios de salud, para poder brindar la atención adecuada y segura, ${ }^{11,35,36}$ por lo que la inversión de sus consultorios ha sido mayor que antes 
y los pacientes resienten estos incrementos, siendo una de las causas por las cuales han dejado de asistir con normalidad.

Además, ha sido necesario reforzar e implementar estrategias de bioseguridad en la propagación del virus en los centros de salud dental, cambios frecuentes en el equipo de protección personal e instauración de protocolos de protección, lo que ocasiona variaciones en costos. La toma de decisiones por parte de los administradores de salud debe contemplar y estimar la consignación de un uso equitativo y racional de los recursos financieros, ya que la pandemia ha provocado una crisis en la actividad económica mundial. ${ }^{37}$

El impacto económico negativo global que ha generado esta pandemia se ha estimado en 30-100 billones de dólares, particularmente por el efecto que ha tenido sobre las industrias y el turismo. ${ }^{38}$ Por lo que se dice que esta pandemia seguirá produciendo pérdidas aún mayores, ante lo cual ya es inminente una depresión en la economía. ${ }^{39}$ Este impacto económico crea un desafío a los odontólogos, ya que dicha depresión económica está afectando a la economía de las personas en todos los niveles y en muchos aspectos, impidiendo que los pacientes tengan la misma capacidad o disponibilidad monetaria y hacer uso de los servicios de odontología, ya que si anteriormente eran de difícil acceso, ahora son más por la pandemia. ${ }^{1,3,40}$

Por todo esto, los odontólogos tienen ahora que invertir más dinero en la adquisición de equipo de protección para el personal que labora dentro de los consultorios $y$, además, proveer a los pacientes y visitantes este tipo de equipo mínimo requerido para poder entrar a sus negocios, haciendo así un círculo de gastos extras. ${ }^{7,11}$

A la fecha, esta pandemia ha ocasionado consecuencias financieras y clínicas que han afectado en la utilización de los servicios de salud, al grado de no ir, posponer o cancelar las citas dentales, repercutiendo en cascada en la economía de los profesionales de la salud oral. ${ }^{3}$

Afortunadamente, hoy en día se cuenta con la telemedicina, la cual ha ayudado a que los ingresos del personal odontológico no se vean disminuidos en su totalidad. Durante una década completa, en Estados Unidos, la telemedicina no había alcanzado la gran popularidad que adquirió en cuestión de meses debido a la pandemia. Sin embargo, la teleodontología no equivaldría nunca a la cantidad de horas laboradas en una clínica de manera presencial, pues vía teleodontología se puede atender de tres a cinco pacientes al día, mientras que presencialmente esa cantidad resultaría mínima. Igualmente, algunos pacientes no tienen acceso a la tecnología o a una red de
Internet, o simplemente, no se sienten muy cómodos con su uso y prefieren no consultar en absoluto, a tener que averiguar la manera de hacer la consulta digitalmente. ${ }^{21}$

También en América, un estudio peruano analizó con detalle ciertos aspectos del ámbito odontológico, entre ellos, que la cantidad de pacientes atendidos sea muy limitada a como lo era previamente. Debido al periodo de ventilación que debe realizarse entre paciente y paciente de aproximadamente 3-4 horas, aunado a la desinfección de las áreas del consultorio, lo que limita mucho la cantidad de pacientes que se pueden atender al día, disminuyendo los ingresos del dentista. Varios odontólogos declararon que la restricción de atender solamente urgencias dentales, no genera los mismos ingresos que se tendrían regularmente, puesto que los tratamientos de urgencia suelen ser sencillos y relativamente económicos; aclarando que sus ingresos se han visto mermados de 20 a $100 \%$ aproximadamente. Asimismo, las restricciones en los viajes y traslados dentro y fuera de los países han ocasionado un desequilibrio en consultorios donde se subcontratan especialistas de otras ciudades para atender a los pacientes, ya que al no poder viajar con seguridad, han decidido dejar de hacerlo, o bien, han sido despedidos, quedando sin ingresos y los pacientes sin poder recibir sus respectivos tratamientos. ${ }^{41}$

Un lado positivo para la atención médica y odontológica es que algunos de los cuidados o tratamientos que los pacientes han estado aplazando, los tendrán que recibir de igual manera más adelante. Por ejemplo, las visitas de atención primaria pospuestas pueden reprogramarse para el próximo verano. Si es así, el personal médico y odontológico que actualmente se encuentra sin laborar, podrá recuperarse económicamente más adelante, conforme todo vaya regresando a la verdadera normalidad. Lo cual no aplica de la misma manera para otras industrias como la restaurantera y de entretenimiento, que quizás nunca recuperen los servicios que no se brindan ahora. ${ }^{42}$

Por otro lado, el impacto de esta pandemia también se da en la salud mental de las personas y varía según el género, nivel educativo y el confort percibido en su hogar mientras vive en confinamiento. Estos aspectos emocionales suelen ser puntos centrales a considerar ante la pandemia del COVID-19. Es así que estas dimensiones, como su impacto emocional y económico, son subjetivas y diferentes entre los distintos grupos sociales, teniendo que ser considerados para la planeación de políticas públicas y no son la excepción las políticas de atención en los consultorios odontológicos para poder afrontar esta pandemia y no afectar su economía y salud en general. ${ }^{39,40}$ 
Con relación a los sentimientos originados por esta pandemia están principalmente el miedo, la incertidumbre y la angustia. Se ha observado que quienes dicen tener menos comodidad en sus hogares, sienten más miedo frente al COVID-19 que los que tienen mayor comodidad, este miedo los invade al grado de afectar su salud y su economía. La incertidumbre, un sentimiento vinculado a consecuencias sociales y económicas que aparentemente se anteponen al aislamiento para la vida cotidiana, social y laboral. En este sentido, algunas personas expresan extrañar a su familia, decir que su economía está afectada porque han perdido su trabajo. Con relación a estas emociones las personas refieren estar angustiados por los familiares que deben presentarse a trabajar, tristeza por los más vulnerables, preocupándose por la economía familiar. ${ }^{38}$

A pesar de lo negativo, también hay consecuencias positivas expresadas en opiniones que se pueden rescatar durante la pandemia. ${ }^{39}$ De entre estas consecuencias positivas resalta la valoración del estado y las instituciones, donde se reconoce el arduo trabajo que hace diariamente el personal de salud y le dan un valor a la salud pública; también aquí se le da un valor al sistema sanitario y científico. Resalta la modificación al modo de producción de un nuevo orden económico. Todos estos aspectos hacen ver la necesidad de entender el impacto económico negativo que causa la pandemia y el efecto negativo o positivo que influye sobre la salud mental de las personas. ${ }^{38,39}$

Ante esta situación sanitaria en México, el Gobierno Federal apostó por una recuperación económica a partir de mantener la inversión pública, otorgar créditos a pequeñas empresas y reforzar las medidas de austeridad dentro de la administración; para llevar a cabo tales acciones, el gobierno propone algunas medidas sociosanitarias, entre las que destacan: suspender todas las actividades no esenciales en el sector público, privado y social, a partir del 30 de marzo de 2020 hasta que el semáforo epidemiológico indique una disminución de la pandemia; las escuelas públicas y privadas en todos los niveles inician el receso durante las mismas fechas; está prohibido realizar reuniones o congregaciones de más de 50 personas; se obliga al lavado frecuente de manos, estornudar o toser aplicando «la etiqueta»; mantener la sana distancia (no saludar de beso, mano ni abrazo); se le pide a la población que viva en México, incluidos los extranjeros, el resguardo domiciliario; el confinamiento obligatorio para los mayores de 60 años o con diagnóstico de hipertensión arterial, enfermedad cardiaca o pulmonar, diabetes, inmunosupresión (adquirida o provocada), embarazadas o puerperio inmediato, independientemente de su actividad laboral. ${ }^{17}$

\section{CONCLUSIONES}

El impacto económico derivado de esta pandemia, sin lugar a dudas, ha afectado la economía de los odontólogos de práctica pública y privada, debido a la adquisición de equipo de protección personal (EPP) e insumos relacionados para el cuidado del paciente y del profesional de la salud, por lo que se puede ver afectado el costo de los tratamientos dentales de los pacientes.

La toma de decisiones de parte de los que administran los consultorios dentales, debe contemplar un uso equilibrado de los recursos financieros, ya que la pandemia ha provocado una crisis en toda actividad económica mundial.

Los servicios de salud deben estar listos para enfrentar las urgencias odontológicas de una forma adecuada, considerando entre sus gastos que la salud de la población es primordial.

\section{REFERENCIAS}

1. Arher A, Patel B, Ruparel NB, Diogenes A, Hargreaves KM. Coronavirus disease 19 (COVID-19): implications for clinical dental care. J Endod. 2020; 46 (5): 584-595. doi: 10.1016/j.joen.2020.03.008.

2. Cevik M, Bamford CGG, Ho A. COVID-19 pandemic a focused review for clinicians. Clin Microbiol Infect. 2020; 26 (7): 842-847. doi: 10.1016/j.cmi.2020.04.02.

3. Provenzano DA, Sitzman BT, Florentino SA, Buterbaugh GA. Clinical and economic strategies in outpatient medical care during the COVID-19 pandemic. Reg Anesth Pain Med. 2020; 45 (8): 579585. doi: 10.1136/rapm-2020-101640.

4. Martí Sánchez D, Fernández Pascual C, Felix Marschall A, Delgado Calva FA, Estébanez Muñoz M, Álvarez Antón S. Aspectos cardiológicos relevantes en la infección COVID-19. RIECS. 2020; 5 (1): 1-13. doi: 10.37536/RIECS.2020.5.1.203.

5. Dingemans AC, Soo RA, Jazieh AR, Rice SJ, Kim YT, Teo LLS et al. Treatment guidance for patients with lung cancer during the coronavirus 2019 pandemic. J Thorac Oncol. 2020; 15 (7): 1119-1136.

6. Jin Y, Yang H, Ji W, Wu W, Chen S, Zhang W et al. Virology, epidemiology, pathogenesis, and control of COVID-19. Viruses. 2020; 12 (4): 372. doi: 10.3390/v12040372.

7. Meng L, Hua F, Bian Z. Coronavirus disease 2019 (COVID-19): emerging and future challenges for dental and oral medicine. J Dent Res. 2020; 99 (5): 481-487. doi: 10.1177/0022034520914246.

8. Organización Mundial de la Salud. Brote de enfermedad por coronavirus (COVID-19): orientaciones para el público. 2020. Disponible en: https://www.who.int/es/emergencies/diseases/novelcoronavirus-2019

9. Peng X, Xu X, Li Y, Cheng L, Zhou X, Ren B. Transmission routes of 2019-nCoV and controls in dental practice. Int J Oral Sci. 2020; 12 (1): 9. doi: 10.1038/s41368-020-0075-9.

10. Baghizadeh Fini $M$. What dentists need to know about COVID-19. Oral Oncol. 2020; 105: 104741. doi: 10.1016/j. oraloncology.2020.104741.

11. Bermúdez-Jiménez C, Gaitán-Fonseca C, Aguilera-Galaviz L. Manejo del paciente en atención odontológica y bioseguridad del personal durante el brote de coronavirus SARS-CoV-2 (COVID-19). Rev ADM. 2020; 77 (2): 88-95. doi: 10.35366/93101.

12. Odeh ND, Babkair H, Abu-Hammad S, Borzangy S, Abu-Hammad A, Abu-Hammad O. COVID-19: present and future challenges for 
dental practice. Int J Environ Res Public Health. 2020; 17 (9): 3151. doi: 10.3390/ijerph17093151.

13. Passarelli PC, Rella E, Manicone PF, Garcia-Godoy F, D'Addona A. The impact of the COVID-19 infection in dentistry. Exp Biol Med (Maywood). 2020; 245 (11): 940-944. doi: $10.1177 / 1535370220928905$.

14. Chamorro-Petronacci C, Martin Carreras-Presas C, Sanz-Marchena A, A Rodríguez-Fernández M, María Suárez-Quintanilla J, RivasMundiña B et al. Assessment of the economic and health-care impact of COVID-19 (SARS-CoV-2) on public and private dental surgeries in Spain: a pilot study. Int J Environ Res Public Health. 2020; 17 (14): 5139. doi: 10.3390/ijerph17145139.

15. Organización de las Naciones Unidas. COVID-19: Ilamamiento urgente para una respuesta a la recesión económica desde los derechos humanos. Experto independiente de la ONU. Ginebra: ONU; 2020. Recuperado de: file://C:/Users/fclsa/Desktop/covid-econom\%C3\%8Da/20200414 IEDebt_urgent_appeal_COVID19_sp.pdf

16. Bong $\bar{C} L$, Brasher $C$, Chikumba $\mathrm{E}$, McDougall R, Mellin-Olsen J, Enright A. The COVID-19 pandemic: effects on low- and middleincome countries. Anesth Analg. 2020; 131 (1): 86-92. doi: 10.1213/ANE.0000000000004846.

17. Organización de las Naciones Unidas para la Alimentación y la Agricultura. La recesión económica mundial causada por la COVID-19: evitar el hambre debe ser un objetivo central del estímulo económico. Italia: FAO; 2020. Recuperado de: file://D:/ covid-economia/CA8800ES.pdf

18. Chávez TM, Castro-Chávez C. Desafíos de la odontología frente a la pandemia del COVID-19. Int J Odontostomat. 2020; 14 (3): 325-326.

19. Schwendicke F, Krois J, Gomez J. Impact of SARS-CoV2 (COVID-19) on dental practices: economic analysis. J Dent. 2020; 99: 103387. doi: 10.1016/j.jdent.2020.103387.

20. Basque Trade investment. Informe impacto de la COVID-19 en México. Grupo spri TALDEA. [Consultado el 19 de diciembre del 2020]. Accesible en: file:///C:/Users/fclsa/Desktop/covideconom\%C3\%8Da/200505-Mexico-Informe-COVID19.pdf

21. Rubin R. COVID-19's crushing effects on medical practices, some of which might not survive. JAMA. 2020; 324 (4): 321-323. doi: 10.1001/jama.2020.11254.

22. Isiekwe IG, Adeyemi TE, Aikins EA, Umeh OD. Perceived impact of the COVID-19 pandemic on orthodontic practice by orthodontists and orthodontic residents in Nigeria. J World Fed Orthod. 2020; 9 (3): 123-128. doi: 10.1016/j.ejwf.2020.07.001.

23. Alradhawi M, Shubber N, Sheppard J, Ali Y. Effects of the COVID-19 pandemic on mental well-being amongst individuals in society- A letter to the editor on "The socio-economic implications of the coronavirus and COVID-19 pandemic: A review". Int J Surg. 2020; 78: 147-148. doi: 10.1016/j.ijsu.2020.04.070.

24. Bhanushali P, Katge F, Deshpande S, Chimata VK, Shetty S, Pradhan D. COVID-19: changing trends and its impact on future of dentistry. Int J Dent. 2020; 8817424. doi: 10.1155/2020/8817424.

25. Nicola M, Alsafi Z, Sohrabi C, Kerwan A, Al-Jabir A, losifidis C et al. The socio-economic implications of the coronavirus pandemic (COVID-19): A review. Int J Surg. 2020; 78: 185-193. doi: 10.1016/j.jjsu.2020.04.018.

26. Bescos R, Casas-Agustench P, Belfield L, Brookes Z, Gabaldón T. Coronavirus Disease 2019 (COVID-19): Emerging and Future Challenges for Dental and Oral Medicine. J Dent Res. 2020; 99 (9): 1113.

27. Nasseh K, Vujicic M. Modeling the impact of COVID-19 on U.S. Dental spending. USA: American Dental Association; 2020.
28. Izzetti R, Nisi M, Gabriele M, Graziani F. COVID-19 transmission in dental practice: brief review of preventive measures in Italy. J Dent Res. 2020; 99 (9): 1030-1038. doi: 10.1177/0022034520920580.

29. Ma C, Gu J, Hou P, Zhang L, Bai Y, Guo Z et al. Incidence, clinical characteristics and prognostic factor of patients with COVID-19: a systematic review and meta-analysis. medRxiv. 2020. doi: 10.1101/2020.03.17.20037572.

30. Carrillo-Esper R, Melgar-Bieberach RE, Tapia-Salazar M, JacintoFlores SA, Campa-Mendoza AN, Pérez-Calatayud AA et al. Manifestaciones extrapulmonares de la infección por SARS-CoV-2. Cir Ciruj. 2020; 88 (5): 654-663. doi: 10.24875/CIRU.20000363.

31. Ferrazzano G, Ingenito A, Cantile T. COVID-19 disease in children: what dentists should know and do to prevent viral spread. The Italian point of view. Int J Environ Res Public Health. 2020; 17 (10): 3642. doi: 10.3390/ijerph17103642.

32. Elangovan S, Mahrous A, Marchini L. Disruptions during a pandemic: Gaps identified and lessons learned. J Dent Educ. 2020; 84: 1270-1274. doi: 10.1002/jdd.12236.

33. Ghai S. Are dental schools adequately preparing dental students to face outbreaks of infectious diseases such as COVID-19? Eur J Dent Educ. 2020; 84 (6): 631-633. doi: 10.1002/jdd.12174.

34. Lyer P, Aziz K, Ojcius D. Impact of COVID-19 on dental education in the United States. J Dent Educ. 2020; 84 (6): 718-722. doi: 10.1002/jdd.12163.

35. Chigurupati R, Panchal N, Henry AM, Batal H, Sethi A, D'innocenzo $\mathrm{R}$ et al. Considerations for oral and maxillofacial surgeons in COVID-19 era: can we sustain the solutions to keep our patients and healthcare personnel safe? J Oral Maxillofac Surg. 2020; 78 (8): 1241-1256. doi: 10.1016/j.joms.2020.05.027.

36. Van-Doremalen N, Bushmaker T, Morris DH, Holbrook MG, Gamble A, Williamson BN et al. Aerosol and surface stability of SARS-CoV-2 as compared with SARS-CoV-1. N Engl J Med. 2020; 382 (16): 1564-1567. doi: 10.1056/NEJMc2004973.

37. Wanderley Y, Oliveira R, De Freitas L, Gomes EH, Baldo AML, Barros DF et al. Economic impact of new biosafety recommendations for dental clinical practice during COVID-19 pandemic. Pesqui Bras Odontopediatria Clin Integr. 2020; 20 (1). doi: 10.1590/pboci.2020.143.

38. Guanche H. COVID-19. Un reto para los profesionales de la salud. Rev Haban Cienc Méd. 2020; 19 (2). Disponible en: http://www. revhabanera.sld.cu/index.php/rhab/article/view/3284/2484

39. Johnson M, Saletti L, Tumas N. Emociones, preocupaciones y reflexiones frente a la pandemia del COVID-19 en Argentina. Ciencia y Salud Colectiva. 2020; 25 (1): 2447-2456. doi: 10.1590/1413-81232020256.1.10472020.

40. Fallahi HR, Keyhan SO, Zandian D, Kim SG, Cheshmi B. Being a front-line dentist during the COVID-19 pandemic: a literature review. Maxilofac Plast Reconstr Surg. 2020; 42 (1): 12. doi: 10.1186/s40902-020-00256-5.

41. Pares-Ballasco G, Castro-Rodríguez Y. Repercusiones de la pandemia COVID-19 en los ingresos y egresos económicos del odontólogo general. Odontol Sanmarquina. 2020; 23 (4): 409-418. doi: 10.15381/os.v23i4.19103.

42. Cutler D. How will COVID-19 affect the health care economy? JAMA. 2020; 323 (22): 2237-2238. doi: 10.1001/jama.2020.7308.

Correspondencia:

Francisco Cázares-de León

E-mail: francisco.cazares@udem.edu ORCID: 0000-0002-0481-8956.

Conflicto de intereses: Los autores declaran no tener ningún conflicto de intereses. 